\title{
Development of High Gain GEM Detectors
}

\author{
A.Bressan ${ }^{1}$, L.Ropelewski ${ }^{1}$, F. Sauli ${ }^{1}$ and D. Mörmann ${ }^{2}$ \\ ${ }^{1}$ CERN, Geneva, Switzerland \\ ${ }^{2}$ Inst. Exp. Kernphysik, Karlsruhe, Germany
}

\section{Abstract}

We describe systematic measurements carried out with single and double GEM detectors with printed circuit readout. The maximum safe operating gain has been measured at increasing radiation flux and under exposure to heavily ionizing tracks. Detection efficiency, localization accuracy and cluster size have been measured in a minimum ionizing particle beam. With a suitably configured readout electrode, fast, two-dimensional localization of radiation is demonstrated.

\section{INTRODUCTION}

The gas electron multiplier (GEM) [1] consists of a thin metal-clad polymer foil chemically perforated by a high density of holes. On application of a difference of potential, the mesh acts as an amplifier for electrons released by radiation in the overlying gas volume (Fig. 1). Originally the device was used in conjunction with a micro-strip plate to boost its gain and improve the reliability of the combined detector [2]. Recent developments in the manufacturing technology, and a better understanding of the operating features, have led to the development of GEM devices with proportional gains up and above $10^{4}$, suitable for direct detection of ionization on simple charge-collecting electrodes [3-7]. GEM detectors have been operated in a wide range of conditions and filling gases [8-10].

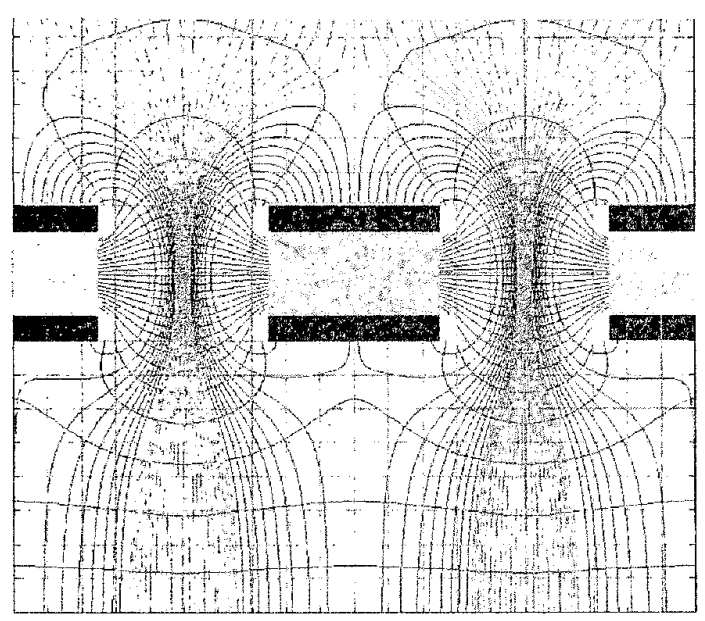

Fig. 1: Field lines and equi-potentials in the GEM channels, computed for typical operating conditions.

\section{TEST BEAM MEASUREMENTS}

Several detectors have been tested in a high-energy beam CERN, of the single and of the double GEM type; in all devices, the conversion region was $3 \mathrm{~mm}$ thick. For the readout we have used high-density amplifier cards based on the PreShape integrated chips, with 45 ns shaping time, connected via twisted pair cables to a gated charge integrating ADCs. For all measurements a gas mixture of argon and carbon dioxide 70-30 was used. Fig. 2 shows the distribution of the total charge, summed over the cluster, for minimum ionizing particles perpendicular to the detector's plane recorded with the single GEM detector in the middle of the operating voltage range. The small peaks on the left represent the electronics noise. With the double GEM, much larger gains are obtained, reaching a signal/noise ratio of 1000 .

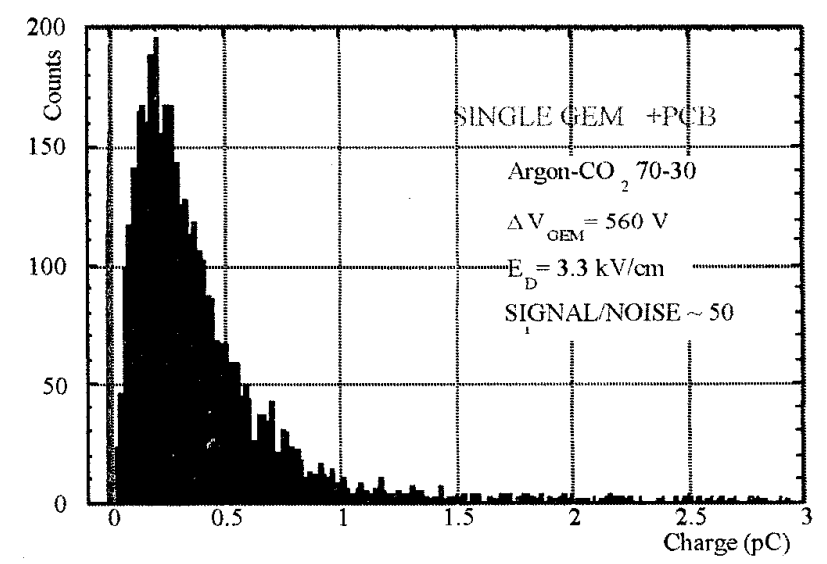

Fig. 2: Total cluster charge for minimum ionizing particles, and noise spectrum measured with the single GEM device.

Making use of the predicted track position provided by a silicon micro-strip telescope, we have computed the efficiency and position accuracy as a function of voltage for the single GEM detector (Fig. 3), and for the double GEM (Fig. 4).

The angular response has been studied by rotating in small increments, the chambers around a vertical axis parallel to the readout strips. Fig. 5 shows, as a function of the angle to the normal, the position accuracy and the most probable value of the total charge for minimum ionizing tracks, for the double GEM. The degradation of accuracy with the incidence angle is a natural consequence of the

$0018-9499 / 00 \$ 10.00$ 다 2000 IEEE 
dispersions due to the primary ionization statistics, and has been observed in all micro-pattern detectors.

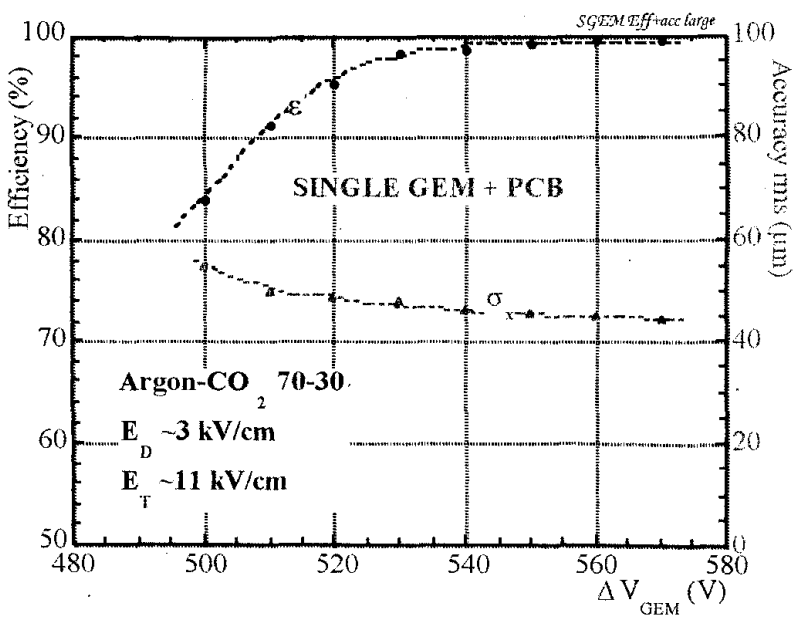

Fịg. 3: Detection efficiency and localization accuracy for minimum ionizing particles in the single GEM detector. The operating gas is argon- $\mathrm{CO}_{2}$ in the volume proportions 70-30.

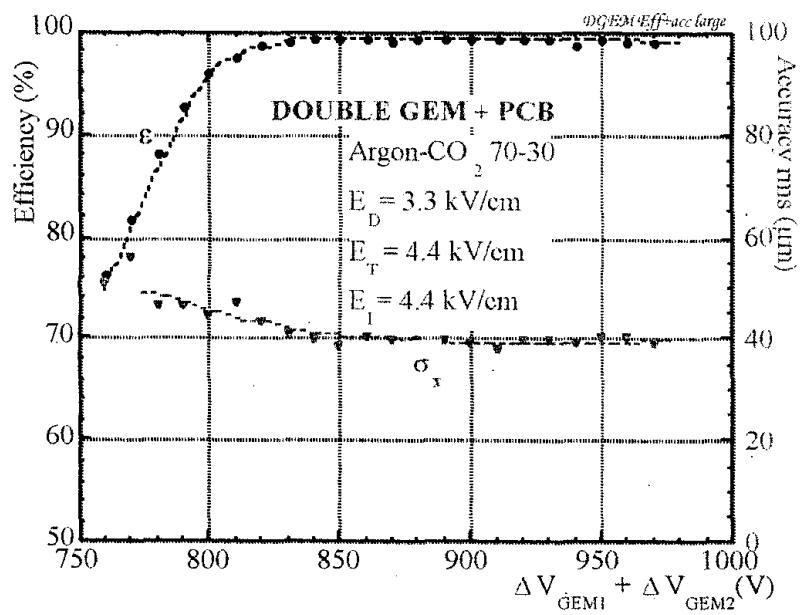

Fig. 4: Detection efficiency and localization accuracy for minimum ionizing particles in the double GEM detector. The two multipliers are operated at equal voltage difference.

\section{Discharge Problems at High Rates AND IONIZATION DENSITIES}

The appearance of discharges is a permanent problem with gas micro-pattern detectors, and has been intensively studied by many authors [11-15]. The general conclusion is that when the total charge in the avalanche exceeds a value between $10^{7}$ and $10^{8}$ electron-ion pairs, the so-called Raether's limit, the enhancement of the electric field in front and behind the primary avalanche induces the fast growth of a long, filament-like charge propagation named streamer; in most cases, this leads to discharge.

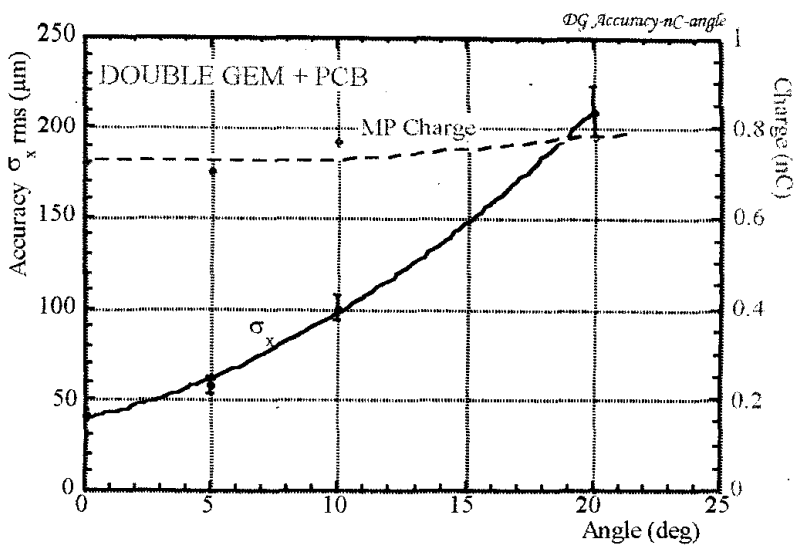

Fig. 5: Angular dependence of the localization accuracy and most probable detected charge in the double GEM detector.

Three major sources of breakdown have been identified: - Spontaneous, in absence of radiation, above a critical voltage: geometry and position-dependent, it reveals the essential role of the manufacturing quality and of local defects.

- Rate-induced: a reduction of the maximum operating voltage due to the onset of discharges at increasing rates.

- Heavily ionizing tracks: exposure to particles with high ionization power results in a considerable decrease of the maximum safe operating voltage. In a thin-gap gas counter, exposure to a particles results in the deposition of $\sim 10^{4}$ pairs per cm; and the Raether limit is reached at gains above $10^{3}$.

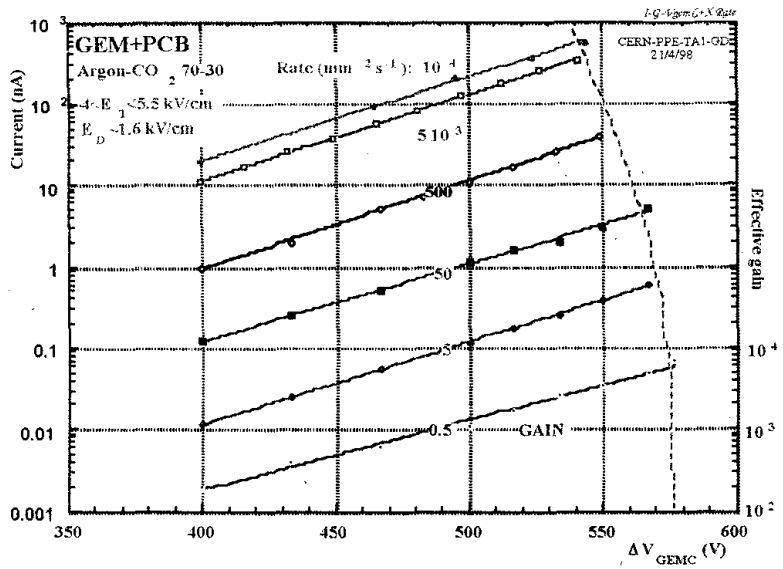

Fig. 6: Anode current as a function of GEM voltage measured at increasing X-ray fluxes (single GEM detector). The dashed line represent the discharge boundary.

Systematic measurements of discharge limits under high-flux $\mathrm{X}$-ray exposure and internal a decays from ${ }^{220} \mathrm{Rn}$ have been performed with a collection of recently developed micropattern detectors; only representative results carried out with GEM devices are reported here. As a filling gas, we have used argon- $\mathrm{CO}_{2}$ in the volume percentage 70-30, a convenient mixture for the use in large detectors, even though 
certainly not the best for obtaining high gains. Fig. 6 shows, as a function of GEM voltage and at increasing rates, the current measured on a group of anode strips whilst irradiating the whole active are of the detector with X-rays. The right scale provides, for the lowest curve, the corresponding values of gain. The dashed curve represents the boundary for stable operation; at the highest flux $\left(10^{4} \mathrm{~Hz} \mathrm{~mm}^{-2}\right)$, the maximum gain is reduced by about a factor of two.

In Fig. 7, one can see the effect of adding the internal a emitter; gain and discharge probability are given as a function of GEM voltage. The requirement of no discharge results in a limiting gain between one and two thousand.

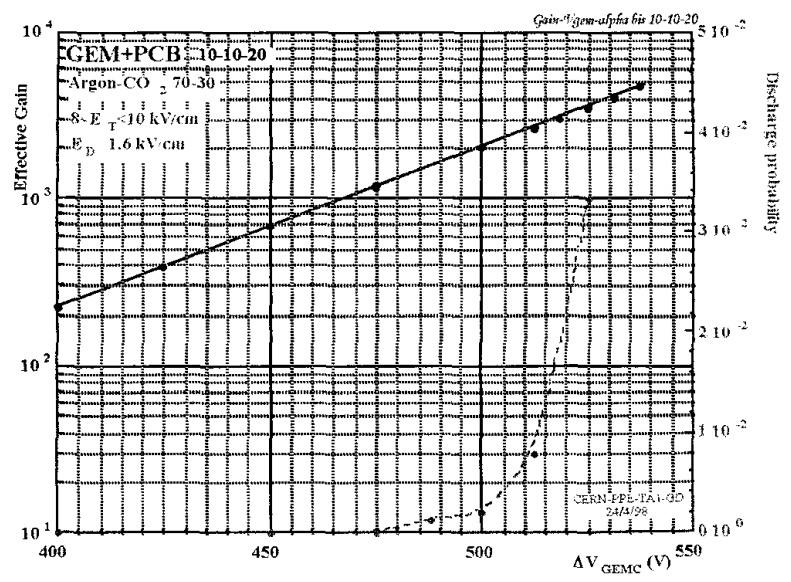

Fig. 7: Alpha-induced discharge probability as a function ov voltage in the single GEM detector.

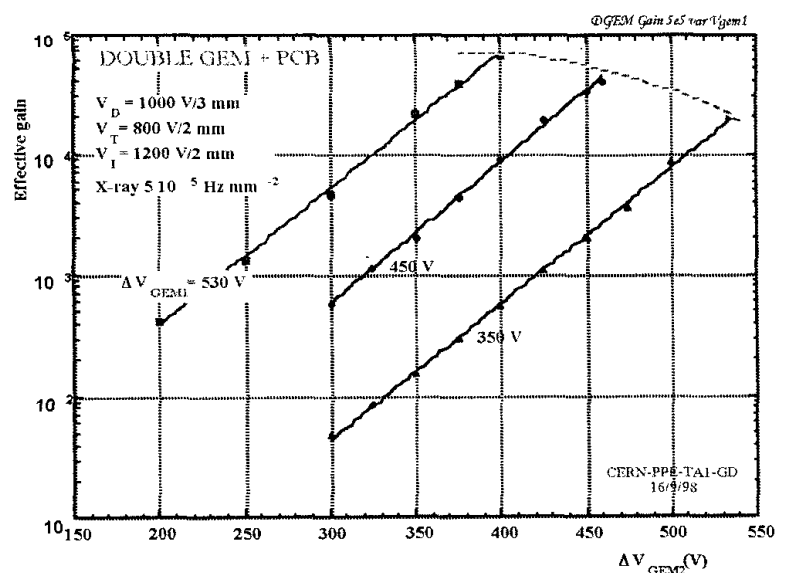

Fig. 8: Maximum gain in the double GEM detector subjected to an X-ray flux of $510^{5} \mathrm{~Hz} \mathrm{~mm}^{-2}$.

Sharing the amplification process between two cascaded devices, a GEM followed by a MSGC or by another GEM, results in a shift upwards by at least an order of magnitude of the maximum gain sustainable. Fig. 8 provides, for the highest irradiation rate $\left(510^{5} \mathrm{~Hz} \mathrm{~mm}^{-2}\right)$ the effective gain as a function of voltage applied; a gain well above $10^{4}$ can be reached. The trend to withstand larger amounts of charge when the second multiplier operates at lower potential supports the presumption of a voltage dependence of the discharge limit.

We have repeated the gain measurement with exposure of the detector to the internal a source; in this case, only the upper boundary of stability before discharge has been recorded (Fig. 9). A gain above $10^{4}$ can be safely reached in a wide range of potentials.

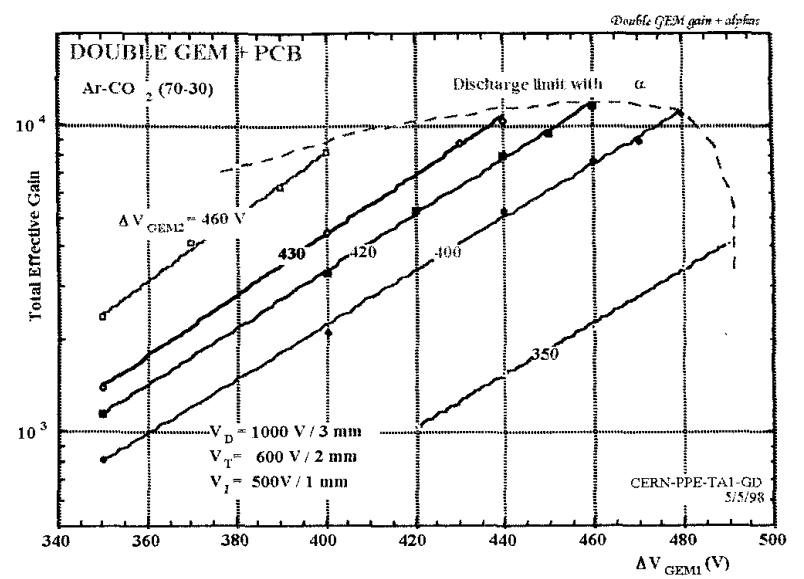

Fig. 9: Discharge limits of the double GEM detector under irradiation of the internal $\alpha$ source.

\section{TWO-DIMENSIONAL READ-OUT}

We have extended the GEM detector principle to include several types of projective two-dimensional readouts; this is done using as pick-up electrode a specially manufactured double-level thin polymer foil, with pads or strips interconnected in various patterns.

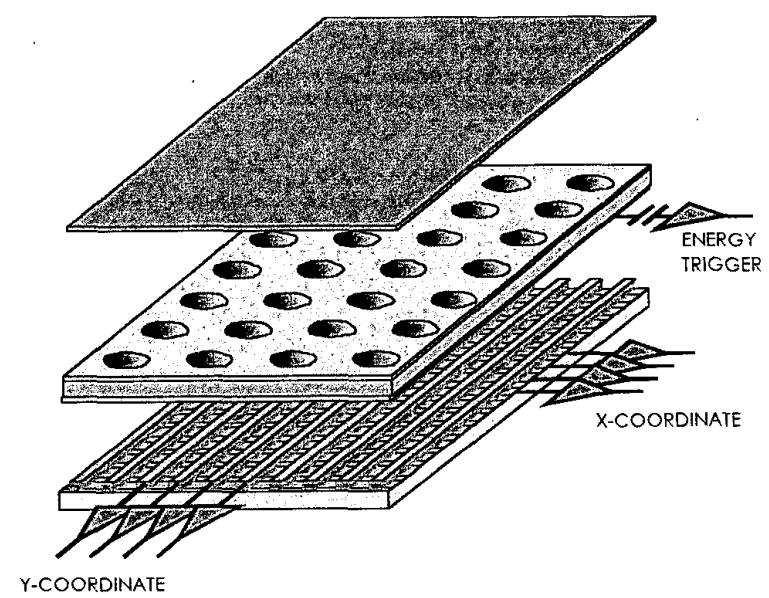

Fig. 10: Two-dimensional GEM read-out. Electrons are collected by the two-levels stripped printed circuit board. The GEM signal can be used for triggering the data acquisition. 


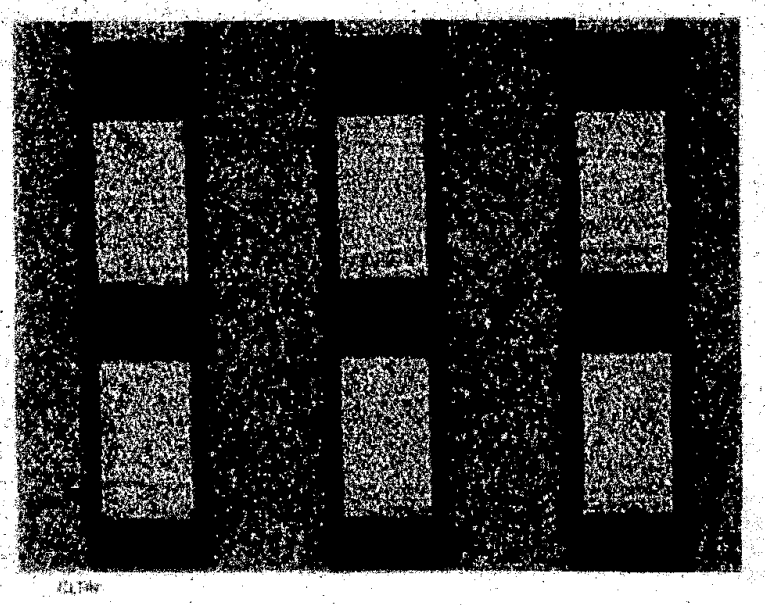

Fig. 11: Close view of the 2-D Cartesian read-out pattern. The pitch is $200 \mu \mathrm{m}$, and strips are 100 and $150 \mu \mathrm{m}$ wide.

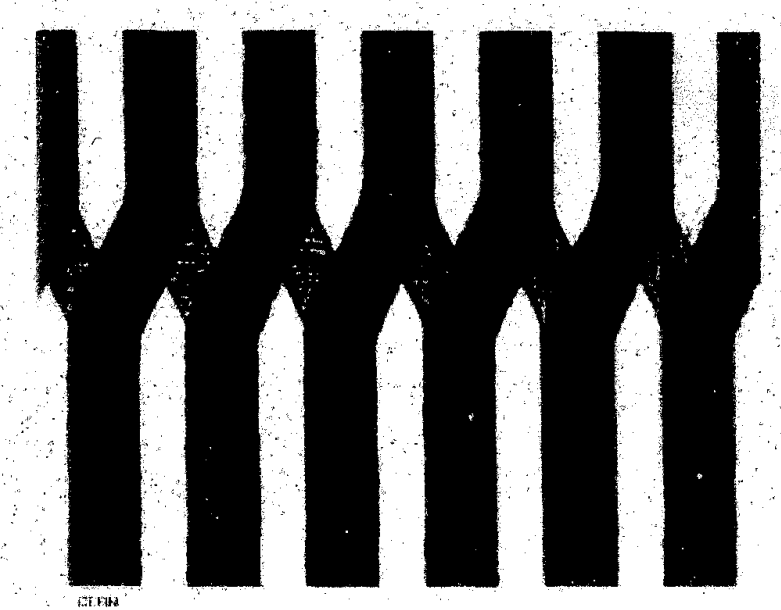

Fig. 12: Close view of the small-angle projective pattern.

In the electron collection mode, both read-out electrodes are kept at ground potential, a substantial advantage as compared to other two-dimensional devices. The method of manufacturing the pick-up electrodes is a direct extension of the one developed for the GEM meshes. Two sets of parallel metal strips are engraved, using conventional printed circuit technology on the two sides of a thin kapton sheet. After gluing the foil on a thin insulating support, the polymer in the interstices between the upper strips is removed with a solvent, opening the bottom layer to charge collection (Fig. 10). The pictures in Fig. 11 and 12 show close views of two read-out patterns successfully manufactured and tested.

Exposing the detector to the X-ray source, we have the induced charge profiles on sets of adjacent strips with gated analogue-to digital converters. The trigger for gating the ADC was provided by the global signal picked up through a HV capacitor from the lower GEM electrode.

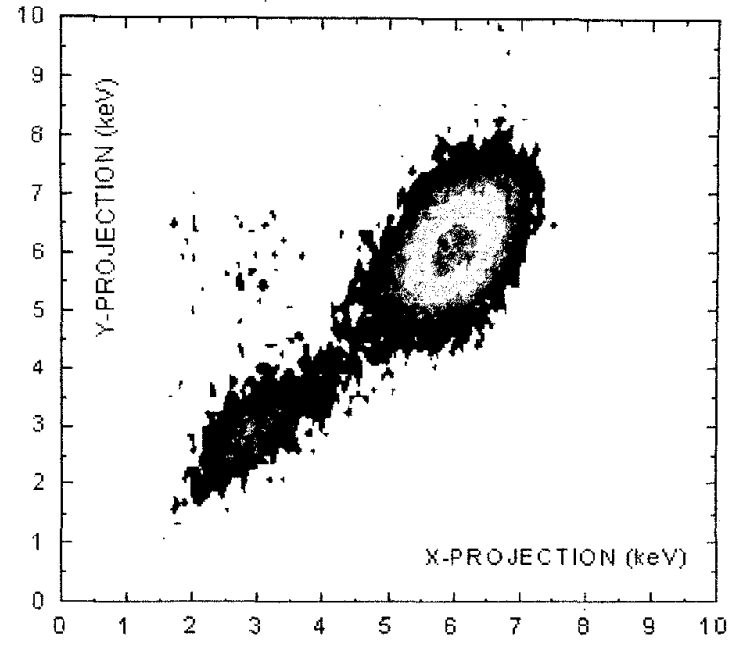

Fig. 13: Charge correlation for $5.9 \mathrm{keV}$ X-rays detected with the two-dimensional Cartesian read-out detector.

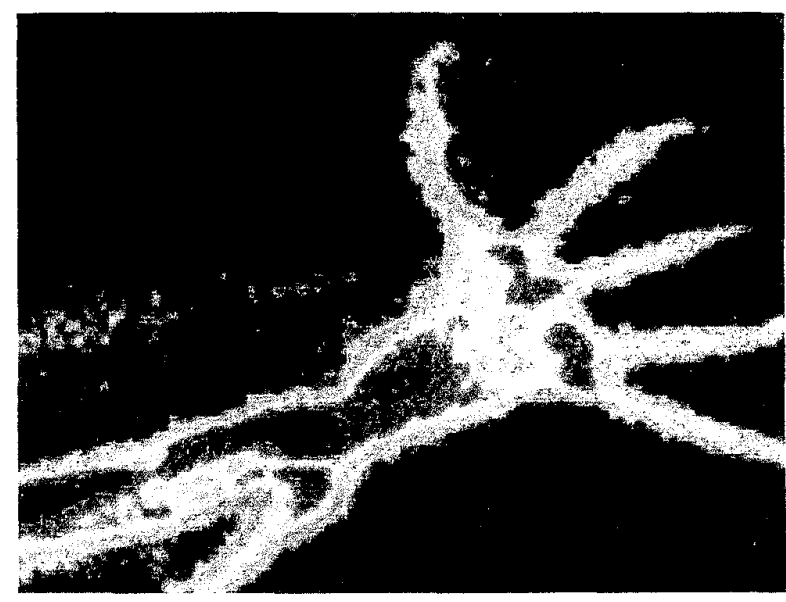

Fig. 14: X-Ray radiography of the foot of a micro-mammal, made with the 2-D GEM detector. The image size is $9 \times 7$ $\mathrm{mm}^{2}$.

Using the Cartesian readout board, we have recorded the charge profile on perpendicular sets of 16 strips, irradiating the detector with a collimated ${ }^{55} \mathrm{Fe}$ source. Fig. 13 shows the correlation plot between the total cluster charge recorded on the two projections; the scales have been normalized to the Xray energy spectrum. The width of the correlation is $17 \%$ fwhm, offering a powerful tool for disentangling multiple events in the detection of charged particles, helped by the wide distribution of the energy loss.

Fig. 14 shows the radiography of the foot of a micromammal realized with the described apparatus, and using an $8 \mathrm{keV} \mathrm{X}$-ray generator as radiation source. The full size of the imaged area is $9 \times 7 \mathrm{~mm} 2$, and the quality of the image demonstrates the rather good resolution of the system. 


\section{REFERENCES}

[1] F. Sauli, GEM: A new concept for electron amplification in gas detectors. Nucl. Instrum. Methods A386 (1997) 531.

[2] R. Bouclier, W. Dominik, M. Hoch, J.C. Labbé, G. Million, L. Ropelewski, F. Sauli, A. Sharma, and G. Manzin, New Observations with the Gas Electron Multiplier (GEM). Nucl. Instrum. Methods A396 (1997) 50-66.

[3] J. Benlloch, A. Bressan, C. Büttner, M. Capeáns, M. Gruwé, M. Hoch, J.C. Labbé, A. Placci, L. Ropelewski, F. Sauli, A. Sharma, and R. Veenhof, Development of the Gas Electron Multiplier (GEM). IEEE Trans. Nucl. Sci. NS-45 (1998) 234.

[4] J. Benlloch, A. Bressan, M. Capeáns, M. Gruwé, M. Hoch, J.C. Labbé, A. Placci, L. Ropelewski, and F. Sauli, Further developments of the Gas Electron Multiplier (GEM), in Nucl. Instrum. Methods. 1998.

[5] A. Bressan, J.C. Labbé, P. Pagano, L. Ropelewski, and F. Sauli, Beam tests of the gas electron multiplier, in Nucl. Instrum. Methods. 1998.

[6] A. Bressan, M. Hoch, P. Pagano, L. Ropelewski, F. Sauli, S. Biagi, A. Buzulutskov, M. Gruwé, A. Sharma, D. Moermann, and G. De Lentdecker, High rate behavior and discharge limits in micro-pattern detectors, in Nucl. Instr. Methods. 1998.

[7] A. Bressan, L. Ropelewski, F. Sauli, D. Mörmann, T. Müller, and H.J. Simonis, Two-dimensional readout in GEM detectors, in Nucl. Instrum. Methods. 1998.

[8] W. Beaumont, T. Beckers, J. DeTroy, V. Van Dyck, O. Bouhali, F. Udo, C. VanderVelde, W. Van Doninck, P. Vanlaer, and V. Zhukov, Studies of an MSGC equipped with a GEM grid as a tracking device, in Nucl. Instrum. Methods. 1998.

[9] A. Bressan, A. Buzulutskov, L. Ropelewski, F. Sauli, and L. Shekhtman, High gain operation of GEM in pure argon, in Nucl. Instr. Methods. 1998.

[10]R. Chechik, A. Breskin, G. Garty, J. Mattout, F. Sauli, and E. Shefer, First results on the GEM operated at low gas pressures, in Nucl. Instrum. Methods. 1998.

[11]T. Beckers, R. Bouclier, C. Garabatos, G. Million, F. Sauli, and L. Shekhtman, Optimization of microstrip gas chamber design and operating conditions. Nucl. Instrum. Methods A346 (1994) 95.

[12] V. Peskov, B.D. Ramsey, J.J. Kolodziejczak, and P. Fonte, Feedback and Breakdowns in Microstrip Gas Counters. Nucl. Instrum. Methods A397 (1997) 243.

[13] V. Peskov, B.D. Ramsey, and P. Fonte, Breakdown features of various microstrip-type gas counter designs and their improvements. IEEE Trans. Nucl. Sci. NS-45 (1998) 244.

[14]B. Schmidt, Microstrip Gas Chambers: recent developments, radiation damage and long term behoviour, in Nucl. Instrum. Methods. 1998.
[15]P. Fonte, V. Peskov, and B.D. Ramsey, Rate and gain limitations of MSGCs and MGCs combined with GEM and other preamplification structures, in Nucl. Instrum. Methods. 1998. 